\title{
Human dorsal anterior cingulate neurons signal conflict by amplifying task-relevant information
}

\author{
R. Becket Ebitz ${ }^{1}$, Elliot H. Smith ${ }^{2}$, Guillermo Horga ${ }^{3}$, \\ Catherine A. Schevon ${ }^{4}$, Mark J. Yates ${ }^{5}$, Guy M. McKhann ${ }^{4}$ \\ Matthew M. Botvinick ${ }^{6}$, Sameer A. Sheth ${ }^{7^{*}}$, and Benjamin Y. Hayden ${ }^{1^{* \dagger}}$
}

1. Department of Neuroscience, Center for Magnetic Resonance Research, and Center for Neural Engineering, University of Minnesota, Minneapolis, MN, 55455, USA

2. Department of Neurosurgery, University of Utah, Salt Lake City, UT, 84132, USA

3. Department of Psychiatry, Columbia University, and New York State Psychiatric Institute, New York, NY, 10032, USA

4. Department of Neurology, Columbia University, NYC,NY,USA 10027

5. Department of Neurological surgery, Columbia University, NYC,NY,USA 10027

6. DeepMind, London, UK

7. Department of Neurosurgery, Baylor College of Medicine, Houston, TX, 77030, USA

$*$ These two authors contributed equally.
+ Lead contact

Keywords: coding dimension, dorsolateral prefrontal cortex, cognitive control, conflict, anterior cingulate cortex

Conflicts of interest: none declared.

Acknowledgements: This work was supported by NIH R01 MH106700, NIH K12 NS080223, NIH S10 OD018211, NIH R01 NS084142, NIH R01 DA038615, the Brain and Behavior Foundation, and the Dana Foundation. Special thanks to Camilla Casadei, David K. Peprah, Yagna Pathak, and Timothy G. Dyster for coordination and data collection efforts. The funders had no role in study design, data collection and analysis, decision to publish, or preparation of the manuscript. 


\section{SUMMARY}

42 Hemodynamic activity in dorsal anterior cingulate cortex (dACC) correlates with

43 conflict, suggesting it contributes to conflict processing. This correlation could be

44 explained by multiple neural processes that can be disambiguated by population firing

45 rates patterns. We used targeted dimensionality reduction to characterize activity of

46 populations of single dACC neurons as humans performed a task that manipulates two

47 forms of conflict. Although conflict enhanced firing rates, this enhancement did not come

48 from a discrete population of domain-general conflict-encoding neurons, nor from a

49 distinct conflict-encoding response axis. Nor was it the epiphenomenal consequence of

50 simultaneous coactivation of action plans. Instead, conflict amplified the task-relevant

51 information encoded across the neuronal population. Effects of conflict were weaker and

52 more heterogeneous in the dorsolateral prefrontal cortex (dlPFC), suggesting that dACC's

53 role in conflict processing may be somewhat specialized. Overall, these results support the

54 theory that conflict biases competition between sensorimotor transformation processes

55 occurring in dACC. 
57

58

59

60

61

62

63

64

65

66

67

68

69

70

71

72

73

74

75

76

77

78

\section{INTRODUCTION}

When faced with conflicting demands for attention or action, we can marshal cognitive resources to maintain effective performance despite this conflict (Shenhav et al., 2013; Botvinick and Braver, 2015; Botvinick and Cohen, 2014; Kerns et al., 2004;

Shenhav et al., 2017). The ability to respond adaptively to conflict is a hallmark of higher cognition, one that allows us to devote the appropriate level of cognitive resources to make good decisions. However, the way in which the brain detects and resolves conflict is a poorly understood aspect of higher-level cognition.

Conflict processing is often associated with the dorsal anterior cingulate cortex (dACC), a region in which conflict alters or increases brain activity (Botvinick et al., 1999; Botvinick et al., 2001 Shenhav et al., 2016). There has been a long-running debate about what effect, if any, conflict has on neuronal computations (Cole et al., 2009). This debate is driven in part by prominent failures to observe conflict correlates at the single unit level, (Amiez et al., 2005; Amiez et al., 2006; Blanchard and Hayden, 2014; Cai and PadoaSchioppa, 2012; Ito et al., 2003; Nakamura et al., 2005). However, more recent studies have shown single neuron correlates of conflict in non-human animals (Ebitz and Platt, 2015; Bryden et al., 2018; Michelet et al., 2015). Most importantly, studies in human $\mathrm{dACC}$ - which lack translational uncertainties associated with model species - provide unambiguous correlates of conflict in both single units and in local field potentials (Sheth et al., 2012; Smith et al., 2019). These results confirm that conflict has direct and measurable neuronal effects but leave unresolved the computations underlying these effects. Here, compare three possibilities, each consistent with recent discoveries, in a 
79 dataset collected in humans performing a conflict task. (Note that these three hypotheses

80 are not necessarily mutually exclusive.)

81 The explicit hypothesis proposes that dACC neurons signal conflict abstractly, in

82 the sense that conflict-related modulations serve the purpose of transmitting information

83 about the presence of conflict - in general - to downstream conflict resolution structures,

84 which implement its resolution. These downstream structures likely include the

85 dorsolateral prefrontal cortex (dlPFC, Johnston et al., 2007; Ma et al., 2019; Smith et al.,

86 2019; MacDonald et al., 2000; Shenhav et al., 2013). In this view, dACC contains either a

87 dedicated, discrete set of neurons specialized for encoding conflict or else its neurons have

88 a distinct population coding axis (sometimes referred to as a dimension, i.e. some linear

89 combination of neuronal responses) that encodes conflict via small, distributed changes

90 across a large number of neurons.

91 The epiphenomenal hypothesis proposes that conflict correlates are the

92 epiphenomenal consequence of the co-activation of neurons that are tuned for different

93 actions (Nakamura et al., 2005) or response predictions (Alexander and Brown, 2011).

94 Epiphenomenal, here, means that correlates of conflict are not driven by computations

95 related to conflict per se, but nonetheless covary with it. This hypothesis was first

96 motivated by the prominent failures to find unit correlates of conflict in a pioneering study

97 of macaque supplementary eye field (SEF), a structure adjacent to dACC (Nakamura et al.,

98 2005). Nakamura and colleagues found that neuronal correlates of conflict in SEF can be

99 explained by co-activation of sets of neurons selective for basic task variables. It is

100 possible that the same ideas may apply to dACC, as goes this hypothesis. 
102 does so by amplifying task-relevant information encoded in dACC neurons. This view is

103 motivated by two observations. First, recent work has amply demonstrated that neurons in

$104 \mathrm{dACC}$ are robustly tuned for a variety of sensory and motor variables (Heilbronner and

105 Hayden, 2016), so the region has all the requisite signals to directly participate in

106 sensorimotor transformations. Second, the ultimate function of conflict processing is not to

107 detect conflict, but to resolve it. One natural way to do so is to amplify task-relevant

108 sensorimotor information at the expense of irrelevant information (Shenhav et al., 2013;

109 Egner and Hirsh, 2005; Botvinick and Cohen, 2014).

110 To arbitrate between these three hypotheses, we examined a large dataset of single 111 neurons recorded in human dACC and, for comparison, a complementary dataset recorded

112 in human dIPFC. Participants performed the multi-source interference (MSIT) task that

113 independently manipulates two forms of conflict, Simon (motor) and Eriksen (perceptual).

114 We find that both forms of conflict modulate responses of single dACC neurons and both

115 tend to increase average firing rates. However, the epiphenomenal hypothesis could not

116 account for neural responses in $\mathrm{AACC}$, and our dimensionality reduction results were more

117 consistent with the amplification hypothesis than with the explicit hypothesis. Our results

118 indicate that conflict robustly enhances the strength of coding of task-relevant

119 sensorimotor information by shifting patterns of population activity along coding

120 dimensions that correspond to the identity of the correct response. This pattern is predicted

121 by the conflict amplification hypothesis. Neurons in dIPFC respond considerably more 
122 weakly and heterogeneously to conflict, suggesting that dACC may have a relatively

123 specialized role in conflict processing. 


\section{RESULTS}

126 patients, dlPFC: $n=9$ patients, see Methods) performing the multi-source interference task

127 (MSIT; Figure 1A-B). This task and its close variants have been widely used to study

128 conflict in humans in studies using both mass action measures and intracranial

129 electrophysiology (Sheth et al., 2012; Smith et al., 2019; Widge et al., 2019a). These data

130 were recorded in human $\mathrm{dACC}$ and dlPFC (Figure 1C). Some of these data come from a

131 set used in a previous publication that focused on local field potentials, which are not

132 relevant to our hypotheses and are not considered here (Smith et al., 2019). The data we

133 study here do not overlap with those used in Sheth et al. (2012), although the tasks are

134 identical.

The MSIT independently manipulates two forms of conflict, either with flanking

136 distractors (Ericksen conflict) or by using the discrepancy between the position of the task-

137 relevant cue and the correct button press (Simon conflict). Response time was slower when

138 any form of conflict was present (Figure 1D; mean z-scored response times: no conflict =-

$1390.29 \pm 0.07$ STE across subjects; any conflict $=0.11 \pm 0.03$ STE; mean within-subject

140 difference $=0.39 \pm 0.1 \mathrm{STE}$; sig. difference, $\mathrm{p}<0.002, \mathrm{t}(2,14)=4.02$, paired $\mathrm{t}$-test $)$. The

141 effects of Simon and Eriksen conflict appeared to be additive; greatest response time

142 slowing occurred when both were present (mean response time for only Simon conflict

143 trials $=-0.03 \pm 0.03 \mathrm{STE}$ across subjects; only Eriksen conflict trials $=0.16 \pm 0.04 \mathrm{STE}$;

144 trials where both forms of conflict were present $=0.28 \pm 0.06 \mathrm{STE}$ ). Further, response time

145 was consistently slower during Eriksen conflict compared to Simon (mean within-subject 
146 difference $=0.19 \pm 0.05$ STE; paired t-test: $\mathrm{p}<0.003, \mathrm{t}(2,14)=3.72)$, suggesting that

147 Ericksen flankers were slightly more effective at driving conflict in this task.

149

150

151

152

153

154

155

156

157

158

159

160

161

162

163

164

165

166

167

168

169

170 a)

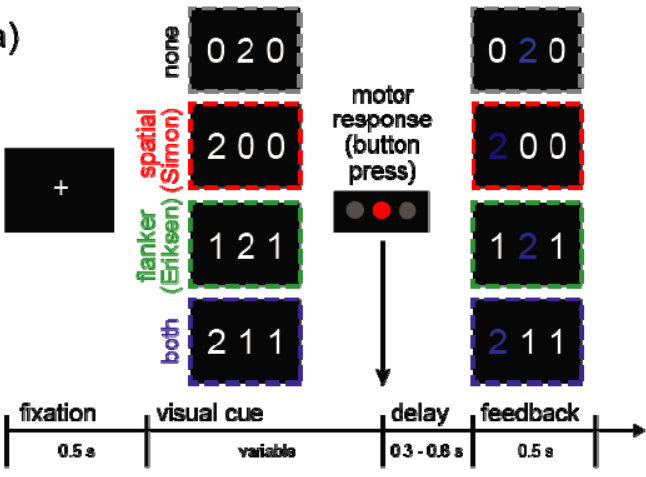

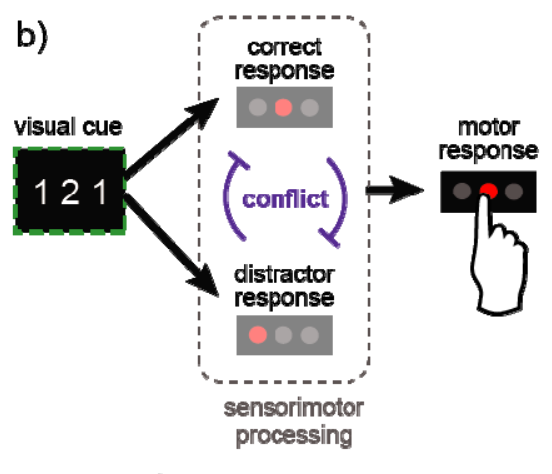

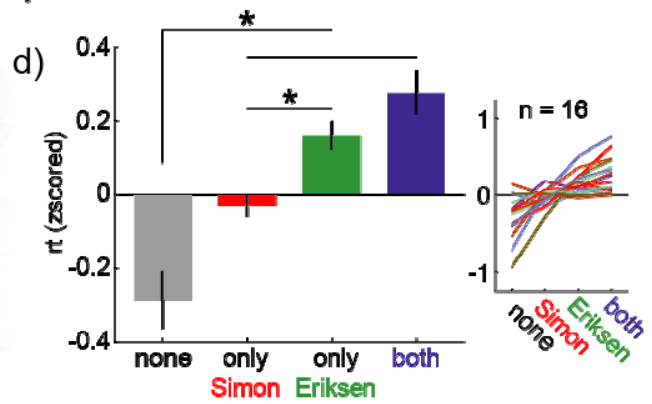

c)

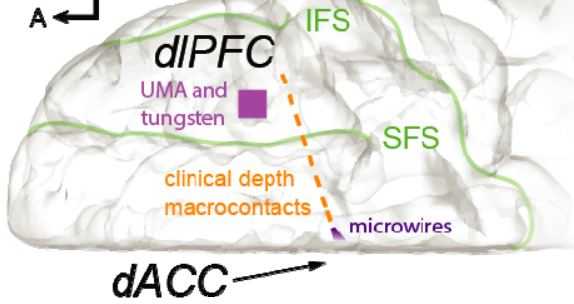

Figure 1. MSIT task and anatomy: A. Structure of the multi-source interference task (MSIT). The subject sees a visual cue consisting of 3 numbers and has to identify the unique number with a button push. The "correct response" is the left button if the target is 1 , middle if 2 , right if 3 . Four example cues are shown here, and in each case, the target is " 2 " and the middle button is the correct response. This is most obvious for the first cue ("none"), where there is no conflicting information. In the other three examples, conflicting information makes the task more difficult. First, incongruence between the location of the target number in the 3-digit sequence and location of the correct button in the 3-button pad produces spatial (Simon) conflict (orange). Second, the distracting presence of numbers that are valid button choices ("1", "2", "3") produces flanker (Eriksen) conflict (green). Trials can also simultaneously have both types (blue). B. The visual cues are associated with one or more sensorimotor responses. Every cue has a correct response, meaning the button press that corresponds to the unique target. Cues can also have one or more distractor responses, meaning the button press that corresponds to task-irrelevant spatial information (Simon) or flanking distractors (Eriksen). If and only if the correct response and distractor response do not match, then the cue causes conflict because only one button response can ultimately be chosen. C. Diagram of the intracranial implant including a stereotactically placed intra-cerebral depth electrode with macroelectrodes (blue squares) along the shaft from dIPFC to dACC and microwire electrodes (orange star) in dACC. A, anterior; 
171 L, lateral; CS, central sulcus; SFS, superior frontal sulcus; IFS, inferior frontal 172 sulcus. The UMA and tungsten microelectrode recoding locations are schematized 173 as a purple square on the surface of dIPFC. D. The average (mean) response 174 times across subjects in each of the four task conditions and (right) the mean 175 response times within each subject. Bars = standard error across subjects.

176

177

178

179

180

181

182

183

184

185

186

187

188

189

190

191

192

193

194

195

196

\section{Encoding of conflict in single neurons in $\mathrm{dACC}$}

We recorded from 145 dACC neurons from 6 human patients. Because our previous investigations show that neural responses can be relatively long-lasting in dACC

(Hayden et al., 2011), we chose a full-trial epoch analysis approach (specifically, a 3 second epoch starting at trial onset, roughly the duration of the trial). Note that we chose this analysis epoch before beginning data analysis. Example cells showing changes in firing rate associated with conflict are shown in Figure 2A and Figure 2B.

Across the population of dACC neurons, activity was higher on Ericksen conflict trials than on no conflict trials (Figure 2A; t-test for all neurons on all trials, $\mathrm{p}<0.03$; mean increase $=0.022 \mathrm{z}$-scored spikes/s $\pm 0.01 \mathrm{STE}$ ). A small number of individual neurons also had different activity levels on Eriksen conflict and no conflict trials $(8.2 \%$, $\mathrm{n}=12 / 145$ neurons, within-cell t-test). This proportion is slightly greater than chance $(\mathrm{p}<$

0.04, one-sided binomial test). In all but one of these neurons, conflict increased firing (significant positive bias, $\mathrm{p}<0.0005$, one-sided, binomial test; mean increase in these cells $=0.30 \mathrm{z}$-scored spikes/s $\pm 0.06 \mathrm{STE}$ ).

Simon conflict was also associated with an increase in activity across the population of dACC neurons, although this increase was not statistically significant

(Figure 2B; $\mathrm{p}<0.06$, mean increase $=0.0007, \mathrm{z}$-scored spikes $/ \mathrm{s} \pm 0.0004$ STE $).$ Overall, $10.3 \%(n=15 / 145)$ neurons had significantly different firing rates between Simon and no- 
197 conflict trials. This proportion is greater than chance $(\mathrm{p}<0.003$, binomial test). However,

198 the sign of conflict encoding in these cells was nearly even (8/15 showed increasing

199 activity; mean increase in these cells $=0.06 \mathrm{z}$-scored spikes/s $\pm 0.09 \mathrm{STE})$. This result

200 indicates that, while dACC neurons do encode Simon conflict, the effect is not strongly (if

201 at all) directional, unlike the positive bias we observed for Eriksen conflict (see above).

202 The largest increase in activity occurred on trials that induced both Simon and

203 Ericksen conflict. Model comparison revealed that the effects of Simon and Ericksen

204 conflict were essentially equivalent and, again, additive across the population of neurons

205 (Figure 2C; Table S1). An additive model was a better fit to the data than other, more

206 flexible models (all BIC weights $<0.02$; sig. additive term: $\beta_{1}=0.033$, $\mathrm{p}<0.003$; see

207 Methods). Thus, though Simon conflict was perhaps more weakly encoded in dACC than

208 Ericksen conflict, regardless of its source, conflict mostly increased the activity of dACC

209 neurons and the effects of Simon and Ericksen conflict were additive.

210 We recorded responses of 378 neurons in dlPFC from 9 patients. In contrast to

211 dACC, we observed little modulation by either form of conflict in dlPFC. Across dlPFC

212 neurons, activity was not higher during Ericksen conflict trials, compared to no-conflict

213 trials $(\mathrm{p}>0.5$, paired t-test; mean increase $<0.0001 \mathrm{z}$-scored spikes/s $\pm 0.0002 \mathrm{STE})$.

214 Average firing rate was higher during Simon conflict, but the effect size was very small (p

$215<0.005 ;$ mean increase $=0.0005$ z-scored spikes $/ \mathrm{s} \pm 0.0002 \mathrm{STE})$. Both effect sizes were

216 significantly smaller than the corresponding effects measured in dACC $(\mathrm{p}<0.001$, $\mathrm{t}$-test).

217 The number of individual neurons that showed individual conflict-related modulation did

218 not significantly exceed the expected false positive rate of 5\% (Ericksen conflict: 5.3\%, 
n=20/378; Simon conflict: $2.9 \%$ n=11/378 neurons). Fewer neurons had any tuning for

either form of conflict in dlPFC, compared to dACC ( $\mathrm{p}<0.05$; compare dlPFC: $7.9 \%$, n=30/378 cells; dACC: $17.9 \%, 26 / 145$ cells; two-sample proportion test, pooled variance).

222 Thus, while conflict responses in dACC were weak, they were larger in dACC than in 223 dlPFC, and responses were more consistently positive in dACC than in dlPFC.
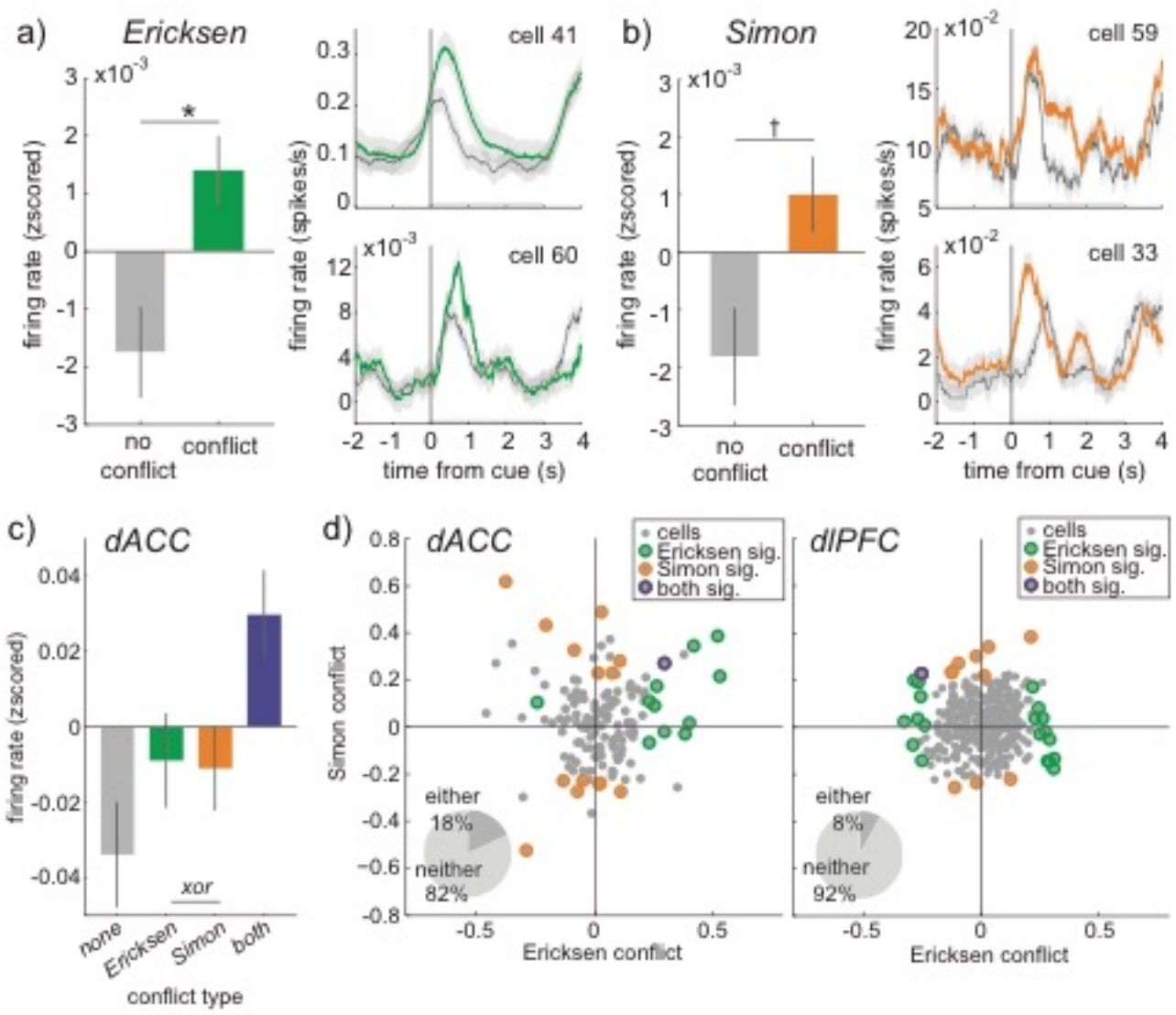

Figure 2) Additive effects of conflict at the population, but different conflict effects in single neurons. A) Left, Average firing rate across all neurons recorded in dACC during Ericksen conflict. Bars $=$ STE, ${ }^{*} p<0.05, \uparrow p<0.1$. Right, Two example cells on no-conflict (gray) and Ericksen conflict trials (red). Ribbons = STE. B) Same as A, for Simon conflict (green). C) Additive effects of each type of conflict. D) Distribution of Simon and Ericksen conflict effects within single neurons in dACC (left) and dIPFC (right). Circled neurons respond significantly $(p<0.05)$ to the highlighted form of conflict $(r e d=$ Ericksen; green $=$ Simon; blue = both). 


\section{Simon and Ericksen conflict tend to affect distinct pools of neurons}

We next considered whether neurons in dACC carry an abstract conflict signal, that

is, one that indicates the presence of conflict, regardless of its source. If dACC detects

243 correlation between the modulation indices for Simon and Ericksen conflict $(r=0.05, p>$

244 0.5; Figure 2D). Furthermore, the population of cells whose responses were significantly

245 affected by Eriksen conflict was almost entirely non-overlapping with the population

246 significantly affected by Simon conflict (specifically, only one cell was significantly

247 modulated by both). The proportion of co-activated dACC neurons was not substantively

248 different from what we observed in dlPFC $(n=1 / 378$ cells significant for both forms of

249 conflict in dlPFC; no difference in proportions, $\mathrm{p}>0.4$, two-sample proportion test with

250 pooled variance). The correlation between Simon and Ericksen conflict responses in dlPFC

251 neurons $(r=0.06, p>0.2)$ also closely matched the values found in dACC. Thus, we found

252 no evidence that dACC neurons uniquely carried some abstract conflict signal. In other

253 words, our evidence does not support the idea that dACC carries conflict-related

254 information that is non-specific to the type of conflict. 
259 of co-activating pools of neurons tuned for different stimuli and/or action plans. This idea

260 was originally proposed by Nakamura et al. (2005). For simplicity, we use the term

261 "response tuning" to indicate selectivity for the sensorimotor responses that were required

262 for the task ("correct responses"), agnostic to whether this tuning was at the level of cue

263 processing, generating the button box response, or the transformation from cue to response.

264 We use the term "distractor response", to refer to the conflicting sensorimotor response

265 indicated by the conflicting cues.

266 Nakamura's epiphenomenal hypothesis predicts that there are separate pools of

267 neurons corresponding to the two conflicting actions, and that conflict increases activity

268 because it uniquely activates both pools. We used ANOVA to jointly estimate the effects

269 of the correct responses, distractor responses, and the conflict between the two on the firing

270 rates of dACC neurons (Figure 3A; see Methods). We found that responses of a significant

271 proportion of neurons were selective for the correct response $(13.1 \% \pm 2.8 \% \mathrm{STE}, \mathrm{n}=$

$27219 / 145$ neurons, this proportion is greater than chance, $5 \%, \mathrm{p}<0.0001$, one-sided binomial

273 test). However, neurons did not encode the distractor response (because we considered

274 tuning for either Ericksen or Simon distractors, the chance level false positive rate was

$2759.75 \%$; percent significant cells $9.7 \% \pm 2.5 \%$ STE, 14/145 neurons, $\mathrm{p}>0.4$, one-sided

276 binomial test against chance). Despite the fact that few neurons encoded the distractor

277 response, a significant proportion of neurons did still signal either Ericksen or Simon

278 conflict $(16.6 \% \pm 2.5 \%$ STE, $\mathrm{n}=24 / 145$ neurons, greater than chance at $9.75 \%, \mathrm{p}<$

279 0.004). Thus, conflict signals occurred more frequently in single neurons than we would 
expect from the epiphenomenal conflict view, where conflict could only emerge in neurons tuned for both correct and distractor responses.

More critically, even in correct response-selective neurons, the preferred correct response rarely matched their preferred Simon/Ericksen distractor response (only $5.3 \%$ of cells matched, $\pm 1.9 \%$ STE, one sided binomial test against chance at $11 \%, \mathrm{p}=1)$. The epiphenomenon hypothesis would predict $100 \%$ match. Moreover, while a very small proportion of cells were response tuned for both correct responses and distractor responses; $2.8 \% \pm 0.1 \%$ STE, 4/145), the majority of conflict-modulated dACC neurons came from a different set $(91.7 \% \pm 0.2 \%$ STE, $n=22 / 24)$. In fact, the majority of conflict-sensitive dACC neurons were not selective for either correct response or distractor responses $(66.7 \%$ $\pm 0.3 \%$ STE, $n=16 / 24)$ - a result that is in direct opposition to the idea that these signals are an emergent consequence of response tuned cells. Thus, not only were response tuned neurons not responsible for the majority of conflict signals in $\mathrm{ACC}$, but the data did not support even the basic premise that there were generic response tuned neurons in dACC. In dlPFC (Figure 3B), conversely, neurons were selective for distractor responses $(14.0 \% \pm 1.8 \%$ STE, 53/378 neurons, greater than chance at $9.75 \%, \mathrm{p}<0.004)$. Like $\mathrm{dACC}$, few neurons were selective for the combination of correct responses and distractor responses $(1.3 \%, 5 / 378$ neurons, sig. greater than chance at $0.5 \%, \mathrm{p}<0.02)$, but in dlPFC these responses matched. Neurons that were tuned for a specific correct response were often tuned to prefer the same Simon/Ericksen distractor response (19\% of cells matched, $\pm 2.0 \%$ STE, one sided binomial test against chance at $11 \%, \mathrm{p}<0.0001)$. Thus, we did see some evidence of generic response tuning in dlPFC, but not in dACC. However, unlike 

$=21 / 378$ ). Ultimately, although the tuning properties of dlPFC neurons were more likely to not dlPFC neurons, were more likely to signal conflict.

307

308

a)

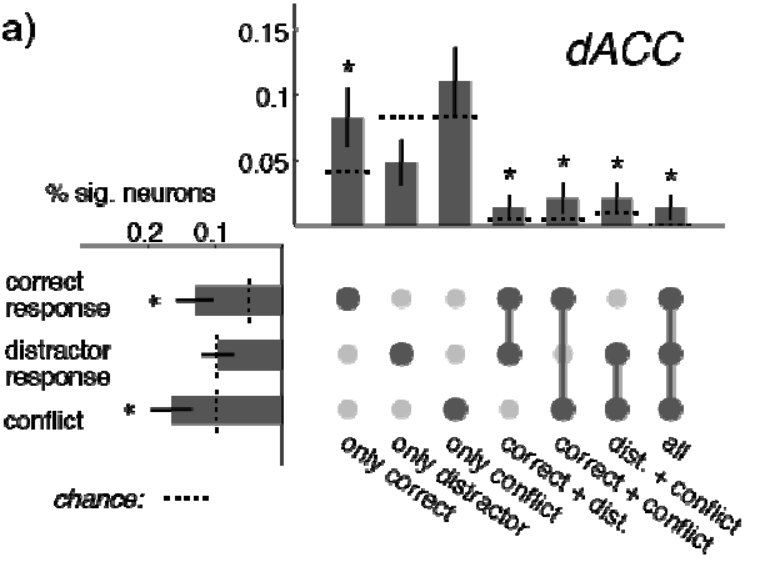

b)

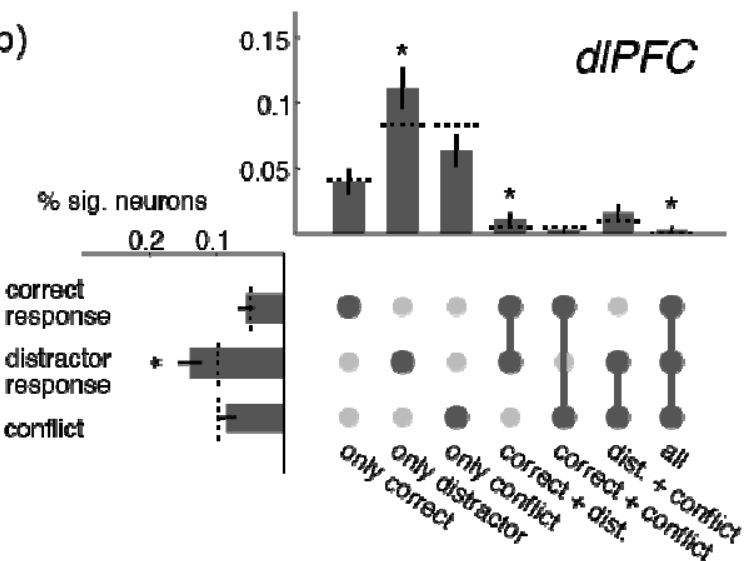

Figure 3) Relationships between task, distractor, and conflict tuning in dACC neurons. A) Percent neurons significantly tuned for task, distractor, conflict (left) and combinations of these variables (top) in dACC. Dotted lines reflect expected false positive rates for each condition. Bars $=$ STE, * $p<0.05$ greater than false positive rate. B) Same as A for dIPFC.

\section{Population analyses can cleanly disambiguate our hypotheses}

At the population level, the three hypotheses make different predictions for neural activity. The explicit hypothesis predicts that there should be either a set of conflictselective neurons or there should be a conflict-selective axis in the population. A population axis is, by our definition here, some linear combination of neuronal firing rates that tracks the presence or absence of conflict, but is distinct from any other parameter the

324 population may encode. Note that the former, stronger prediction (a subset of conflict- 
325 selective cells) would also satisfy the latter, weaker prediction (a conflict-encoding axis),

326 so we focus on the latter prediction to maximize the chances of validating this model. In

327 the epiphenomenon hypothesis, when correct response and distractor responses match

328 (i.e., when there is no conflict), both inputs activate the same set of neurons (Figure $\mathbf{4 A}$,

329 left). When they are in conflict, separate sets of neurons are activated (Figure 4A, right,

330 Nakamura et al., 2005). At the population level, then, the epiphenomenon hypothesis

331 predicts that conflict should decrease the amount of information about the correct response

332 and shift neuronal population activity down along the axis in firing rate space that encodes

333 this response (Figure 4B). Note that net population activity will only increase if conflict

334 increases activity in the distractor response neurons more than it decreases activity in the

335 correct response neurons (Nakamura et al., 2005). As a result, in the epiphenomenal

336 hypothesis, as in the explicit hypothesis, there will be a population axis that selectively

337 encodes conflict, corresponding to the summed activity of all the neurons. However, in the

338 explicit case, this shift will only be in the direction of a unified conflict detection axis,

339 whereas in the epiphenomenal view, it will largely, but not exclusively be along the coding

340 dimensions in firing rate space that discriminate one response from another (Figure 4C

341 and D). The amplification hypothesis, conversely, does not predict a unified conflict

342 detection axis in the population. Instead, it makes a prediction that is exactly contrary to

343 the epiphenomenal view: that conflict should shift population activity along task-variable

344 coding dimensions, but in the opposite direction. That is, conflict is predicted to amplify

345 task-relevant neural responses (Figure 4E). 
347 Conflict amplifies encoding of correct response information dACC

To arbitrate between these hypotheses, we must determine where trials fall along

349 the coding dimensions for each correct response, where the "coding dimensions" are the

350 combinations of neuronal firing rates that best predict the likelihood that the subjects are

351 performing one of the three motor responses. We did this by combining the responses of

352 neurons recorded separately into pseudopopulations (Churchland et al., 2012; Machens et

353 al., 2010; Mante et al., 2013; Meyers et al., 2008; Ebitz et al., 2019) and then using a form

354 of targeted dimensionality reduction to identify the coding dimensions in the population

355 activity (Ebitz et al., 2018; Ebitz et al., 2019; Peixoto et al., 2019; Cunningham and Yu,

356 2014). Briefly, we used multiple logistic regression to identify the linear combinations of

357 neuronal firing rates that encoded specific correct responses, then projected the activity

358 from individual trials onto each coding dimension, that is, into the subspace defined by the

359 coding dimensions corresponding to the three different responses (Figure 4F; see

360 Methods).

361 When population activity was projected into task-coding space, it was easy to

362 predict the current correct response from neural activity (Figure 4G-H; across 1000

363 bootstrapped populations: mean projection onto correct response coding dimension $=1.19$,

$36495 \% \mathrm{CI}=[1.08,1.30]$; mean projection onto other response dimensions $=-0.76,95 \% \mathrm{CI}=$

$365[-0.82,-0.70]$; mean $\mathrm{AUC}=0.97,95 \% \mathrm{CI}=[0.96,0.98])$. However, classification accuracy

366 was even higher for trials with Ericksen conflict than it was for trials without Ericksen

367 conflict (Figure 4I; sig. difference between conflict and no-conflict, $\mathrm{p}<0.02$; conflict,

368 mean $\mathrm{AUC}=0.98,95 \% \mathrm{CI}=[0.97,0.99]$; no conflict, mean $\mathrm{AUC}=0.94,95 \% \mathrm{CI}=$ 
369 [0.91,0.98]; representative population: conflict, mean AUC $=0.996$, correctly classified

$37096.8 \%$ or $122 / 126$ trials, no conflict $\mathrm{AUC}=0.980,87.3 \%$ correct or $55 / 63$ trials, sig.

371 change in correct classification likelihood, $\mathrm{p}<0.04,2$ sample proportion test with pooled 372 variance).

373 The increase in classification accuracy was due to an increase in the projection onto

374 the correct response coding dimension (Figure 4J-K; $\mathrm{p}<0.03$, bootstrap test of the

375 hypothesis that conflict minus no conflict is > 0; all trials: mean difference in projection

376 onto task coding dimension $=0.24,95 \% \mathrm{CI}=[0.02,0.48]$; task 1 trials only: mean $=0.35$,

$37795 \% \mathrm{CI}=[-0.08,0.79] ;$ only task 2 trials: mean $=0.26,95 \% \mathrm{CI}=[-0.12,0.63] ;$ only task 3

378 trials: mean $=0.12,95 \% \mathrm{CI}=[-0.33,0.54])$. Thus, conflict increased the amount of correct

379 response information in populations of neurons through shifting neural representations up

380 the task coding axes, consistent with the amplification hypothesis. These population-level

381 effects of conflict were qualitatively similar to what we observed in dlPFC (sig. difference

382 in classification accuracy between conflict and no-conflict, $\mathrm{p}<0.04$, conflict, mean AUC $=$

$3830.97,95 \% \mathrm{CI}=[0.95,0.99] ;$ no conflict, mean $\mathrm{AUC}=0.92,95 \% \mathrm{CI}=[0.87,0.96]$;

384 increased projection onto correct response coding dimension during conflict, $\mathrm{p}<0.2$, mean

385 difference in projection onto task coding dimension, all trial types $=0.30,95 \% \mathrm{CI}=[0.03$, $3860.58])$. 
a)

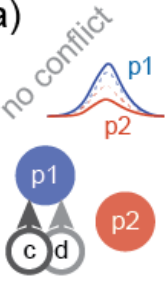

c) emergent

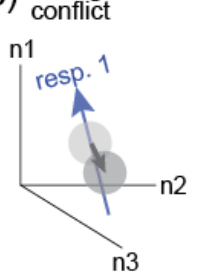

b)

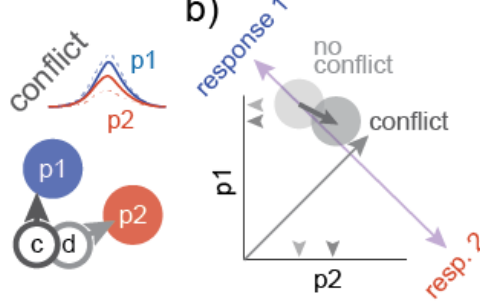

d) $\begin{gathered}\text { conflict } \\ \text { detection }\end{gathered}$

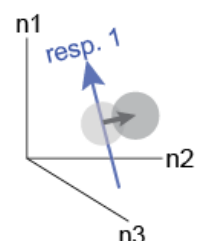

e) $\begin{aligned} & \text { conflict } \\ & \text { amplification }\end{aligned}$

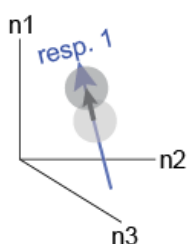

f)

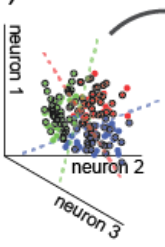
n3

\section{Figure 4) Population-level analyses suggest that dACC conflict signals} amplify task representations. A) Cartoon of the epiphenomenal conflict hypothesis, where separate pools of neurons are tuned for response 1 (p1, blue) and 2 (p2, red). When the correct response is response 1 and there is no conflict, correct response (c) and distractor response (d) information both activate $\mathrm{p} 1$. When there is conflict, distractor information increases p2 activity at the expense of $\mathrm{p} 1$. If conflict increases $\mathrm{p} 2$ activity more than it decreases $\mathrm{p} 1$, total neural activity will be higher during conflict. B) A population view of the epiphenomenal conflict hypothesis. Here, p1 and $\mathrm{p} 2$ activity form the axes of a firing rate space, in which trials are distributed (shaded circles). In this firing rate space, there is a coding dimension that differentiates neural activity for correct response 1 (correct response $=1$, regardless of conflict) from neural activity for the other responses, here response 2 . This coding dimension is $p 1-p 2$ here. In the epiphenomenal hypothesis, conflict decreases p1 activity and increases p2, which would largely shift response 1 activity down along the response coding dimension that differentiates response 1 from other responses. A conflict signal is epiphenomenal if activity also moves above this manifold, along an orthogonal, conflict-detecting axis (here, p1 + p2). C) The epiphenomenal hypothesis predicts that conflict should mostly, though not exclusively, shift activity down response coding dimensions, because it decreases the encoding of the correct response in favor of the distractor response. D) The explicit hypothesis predicts that conflict should largely shift activity along some conflict-detecting dimension that is orthogonal to response coding. E) The amplification hypothesis predicts that conflict should 
amplify the representation of response information-shifting activity up the response coding dimensions. F) Targeted dimensionality reduction to find response coding dimensions in the data. We find the separating hyperplanes that discriminate each response from the other two (left), project individual conflict (black circle) and no-conflict (no outline) trials into the subspace defined by these separating hyperplanes (middle), and measure projections onto the resulting response coding dimensions (right; pale arrows). G) Projections of one representative pseudopopulation onto the coding dimension that corresponds to the correct response that subjects executed on the trial (saturated), or to the other two responses (light). $\mathrm{H}$ ) Distribution of mean projections onto the correct response and the other responses across pseudopopulations (left) and the distribution of $A U C$ values for discriminating the correct response from the other responses based on these projections (right). I) Top: Task classification accuracy from coding dimension projections for conflict and no-conflict trials. One representative pseudopopulation. Bottom: Average AUC values for conflict and no conflict trials across pseudopopulations. J) Projections of conflict and no-conflict trials onto the correct response coding dimensions. $\mathrm{H}$ ) Difference in correct response coding dimension projections between conflict and no conflict trials across pseudopopulations. Bars \pm SEM and box plots illustrate the median, $50 \%$ and $95 \%$ Cl. ${ }^{*}=p<0.05, \dagger=p<0.1$.

\section{No abstract conflict coding axis in the population}

Together, these results support the hypothesis that conflict amplifies neural coding of task variables within dACC. However, these results do not rule out the existence of a unified conflict axis. It thus remains possible that dACC both signal conflict and amplifies encoding of task variables. Therefore, we next asked whether there was a conflict detection axis in the population by examining the representational geometry of task variable and conflict coding dimensions in the region. Just as there are coding dimensions in the population that correspond to the task the subjects were performing, there are coding dimensions that correspond to the presence or absence of conflict during these tasks. In the amplification view, these must be at least partially aligned to the relevant task coding axis

(Figure 5A-B). However, these conflict coding dimensions could also be at least partially 
447 aligned with each other. This would indicate that there is some average conflict coding

448 vector that could be used to decode the presence or absence of conflict, regardless of the

449 task. It would mean there was a conflict detection axis in the dACC population.

450 We found that the conflict coding dimensions for each task were aligned with the

451 task variable coding dimensions, both in the representative population (Figure 5C; more

452 aligned than shuffled data, one-sided permutation test, $\mathrm{p}=0.001$ ) and across all the

453 populations (Figure 5D; one-sided permutation test, $\mathrm{p}=0.003$ ). However, conflict coding

454 dimensions were not aligned with each other (Figure 5E; not more aligned than shuffled

455 data, one-sided permutation test, $\mathrm{p}>0.5$ ). Thus, while average neuronal firing rates tended

456 to be higher when Ericksen conflict was present (Figure 2A) and this same trend was

457 apparent regardless of the correct response (Figure 5F-G; mean difference, correct

458 response $1=0.03 \mathrm{z}$-scored spikes/s, response $2=0.05$, response $3=0.02$ ), there was

459 ultimately no explicit conflict detection axis in the dACC population. Instead, conflict

460 amplified the encoding of the correct response. 
a)

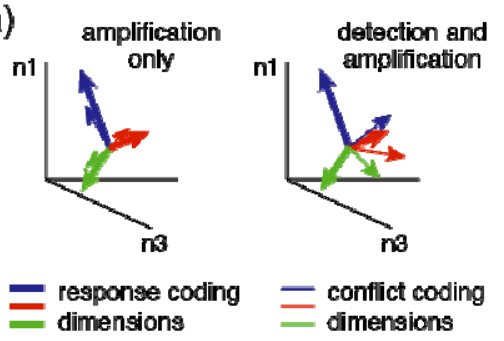

c)
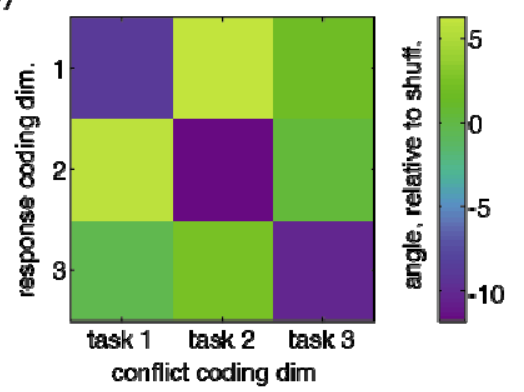

b)

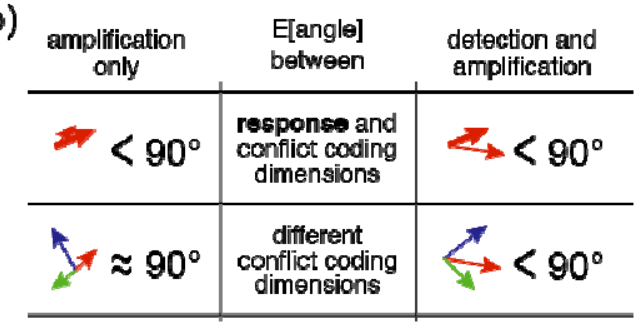

d)

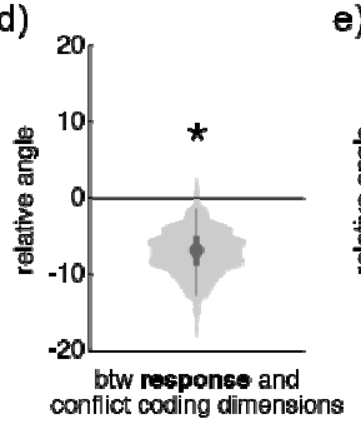

e)

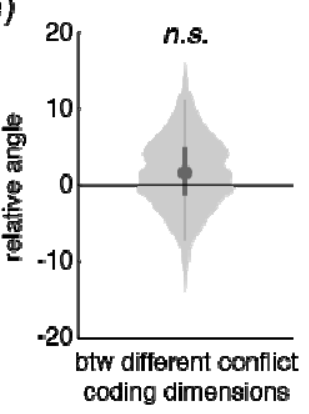

ding dimensions

f)

462
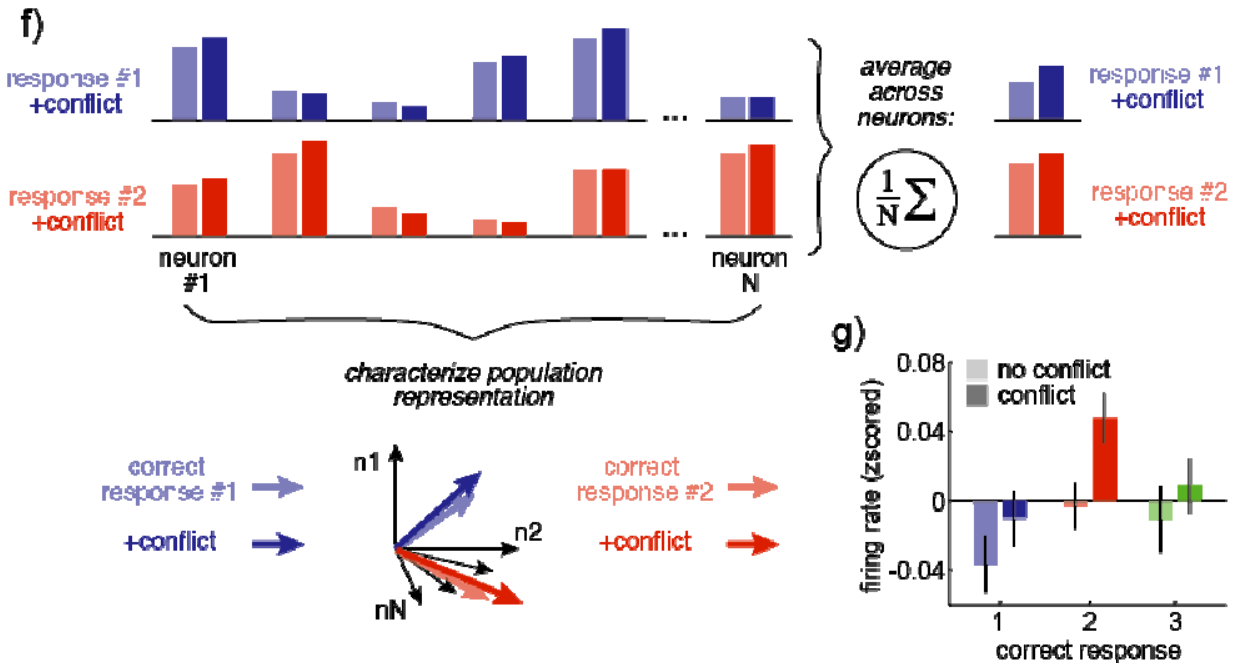

\section{Figure 5) Relationship between task and conflict coding dimensions. A) A} cartoon illustrating possible geometric relationships between the correct response coding dimensions and the population dimensions that encode the presence/absence of conflict during each task. Left) If conflict amplifies correct response information, conflict coding dimensions should be aligned with the matching correct response axes. Right) If $\mathrm{dACC}$ both explicitly detects conflict and amplifies correct responses, then there should be a shared conflict detection axis in the dACC population, which would mean that conflict coding axes will be at least partially aligned with each other. B) Predictions of the two hypotheses illustrated in A. For any amplification to occur, conflict coding dimensions must be somewhat aligned with the matching correct response coding dimensions. However, in the explicit conflict detection view, conflict coding dimensions would also be somewhat aligned with each other. C) The angle between each correct response coding 
477 dimension and conflict coding dimensions in the representative population. The

478 diagonal structure indicates that conflict coding dimensions are aligned with

479 matching response coding dimensions. Angles were normalized by subtracting the

480 mean of label-permuted data, so $0=$ no alignment. D) Distribution of angles

481 between conflict coding dimensions and matched response coding dimensions

482 across populations. E) Distribution of angles between the different conflict coding

483 dimensions. F) A cartoon illustrating the central results. The population of neurons

484 has a heterogenous pattern of activity for each correct response. Conflict

485 modulates these patterns in different ways. Nevertheless, when averaging over

486 neurons, conflict will generally increase activity, regardless of the correct

487 response. However, one can also consider the whole pattern of activity across

488 neurons, here illustrated as a neuron-dimensional vector. In this view, it becomes

489 clear that the pattern of conflict modulation during one correct response is

490 orthogonal to the pattern during another correct response. G) Conflict tended to

491 increase average firing rates across neurons for each correct response condition,

492 despite having orthogonal effects at the level of the pattern of population activity.

493 Bars \pm SEM across neurons. 


\section{DISCUSSION}

We sought to understand the neural basis of conflict processing by examining responses of neurons in human $\mathrm{dACC}$ and dlPFC collected in a conflict task. A previous paper from our team focused on spike-LFP relationships in this dataset and asked very different questions; the present one focuses on single unit activity (Smith et al., 2019). Here we show that the activity of dACC neurons tended to increase when conflict was present, consistent with most studies using mass action measures and with some recent neurophysiological studies (Sheth et al., 2012; Smith et al., 2019; Ebitz and Platt, 2015; Bryden et al., 2018; Michelet et al., 2015). Our major goal was to go beyond correlating neural activity with task variables, and to instead use targeted dimensionality reduction to determine what specific neuronal computations gave rise to this conflict signal. This method allowed us to directly compare and reject two major hypotheses in the literature, which we call the explicit hypothesis and the epiphenomenal hypothesis (Nakamura et al., 2005; Cole et al., 2009; Schall and Emeric, 2010; Mansouri et al., 2017; Cole et al., 2010; Kolling et al., 2016; Shenhav et al., 2016; Stuphorn et al., 2000). Instead, the data supported a third amplification hypothesis, that the effects of conflict are to amplify the encoding of task-relevant information across populations of neurons. Specifically, when conflict was present, the neural representation of the correct task-relevant sensorimotor responses was enhanced at the expense of irrelevant and incompatible responses (cf. Egner and Hirsch, 2005; Pastor-Bernier and Cisek, 2011; Cisek, 2007). Attempts to determine the function of dACC have historically centered on identifying a specific executive role, that is, one that supports or modifies sensorimotor 
516 transformation but is external to and conceptually distinct from it (Paus, 2001; Bush et al.,

517 2000; Ebitz \& Hayden, 2016). This view is at least somewhat inconsistent with the

518 growing literature identifying robust correlates of sensorimotor transformation in the

519 region (e.g. Kennerley et al., 2011; Isomura et al., 2003; Johnston et al., 2007; Gemba et

520 al., 1986; Hillman and Bilkey, 2010; Strait et al., 2016; Azab and Hayden, 2017; reviewed

521 in Heilbronner and Hayden, 2016). That literature both suggests that dACC may have a

522 sensorimotor role in addition to any executive role, and raises the broader question of how

523 executive processes modulate sensorimotor transformations. It may helpful to think of

524 dACC as one layer in a hierarchy of structures that can regulate goal-directed behavior by

525 distributed changes in the gain of sensorimotor transformations (Cisek and Kalaska, 2010;

526 Pezzulo and Cisek, 2016; Yoo and Hayden, 2018; Ebitz \& Moore, 2017; Ebitz et al.,

527 2019). Our results suggest that conflict is one of the executive processes that modulates

528 sensorimotor encodings in this way. Note that conflict also has clear effects on the timing

529 of action potentials, relative to ongoing local field potentials in this region, which may

530 modulate the spiking effects we observed (Smith et al., 2019).

531 Amplification of task-relevant responses could push the system to focus on

532 computations most relevant to the task at hand (Suzuki and Gottlieb, 2013; Finklestein et

533 al., 2019; Ebitz et al., 2019; Ebitz et al., 2018; Egner \& Hirsch, 2005). In this regard, we

534 would draw an analogy between the effects of conflict we report in the prefrontal cortex

535 and the effects of selective visual attention on sensory representations in the ventral visual

536 stream (Desimone and Duncan, 1995; Desimone, 1996; McAdams and Maunsell, 1999).

537 Attention is the enhanced representation of behaviorally-relevant stimuli at the expense of 
538 other stimuli. Representations of stimuli naturally compete for control of behavior, and

539 attention functions to bias this competition towards behaviorally relevant representations.

540 Notably, the competition between representations does not stop at the rostral pole of the

541 temporal lobe, but continues through to the motor system (Cisek and Kalaska, 2010; Cisek,

542 2007; Cisek, 2012). While it is not clear whether the same computations are involved in

543 biasing competition between sensory representations in extrastriate cortex, motor

544 representations in motor cortices, or sensorimotor representations in association cortices, it

545 is clear that there could be a continued benefit in a biasing process that can tip the scales

546 towards favored action at any point throughout the sensorimotor transformation. Further,

547 visual attention may produce shifts in population-level stimulus representations in

548 extrastriate cortex that resemble the shifts that conflict produces in sensorimotor

549 representations in the prefrontal cortex (Cohen and Maunsell, 2010). Thus, it seems

550 prudent to consider the possibility that cognitive processes like conflict may invoke

551 computational processes that resemble those that bias competition between sensory

552 representations in extrastriate cortex (Cisek, 2007; Michelet et al., 2010; Pastor-Bernier

553 and Cisek, 2011; Yoo et al., 2018). In other words, our findings are consistent with the idea

554 that the brain uses conserved computational processes to solve similar problems in

555 different ends of the brain (Yoo and Hayden, 2018; Hunt et al., 2017).

556 Our results highlight the differences between dACC and dlPFC. These two regions

557 are strongly interconnected, and are both strongly implicated in many executive functions.

558 This relatedness does not necessarily imply that they have identical roles, however (Smith

559 et al., 2019; Hunt et al., 2018). Indeed, anatomy and functional studies both motivate the 
560 hypothesis that these regions may have a hierarchical relationship (Shenhav et al., 2017;

561 Miller and Cohen, 2000; MacDonald et al., 2000) as do at least some physiological studies

562 (Hunt et al., 2018). In this hierarchical view, the increase in conflict modulation that we

563 observed in dACC neurons may occur because the region responds to conflict at an earlier

564 and more abstract level of the hierarchy, while dlPFC is less modulated by conflict because

565 it is later and presumably more effector-specific. Of course, the hierarchical view does not

566 require that regions must have strict functional differences, but instead a gradual shift in

567 function along a hierarchy that transforms sensations to actions (Hunt et al., 2017; Yoo and 568 Hayden, 2018).

569 The differences between the Eriksen/flanker and Simon/response conflict effects 570 we report here echo earlier findings from human EEG (Van Veen et al., 2001) and primate 571 neurophysiology (Ebitz and Platt, 2015). These earlier studies report that that conflict

572 encoding can differ depending on whether the conflict is between responses / stimuli (Van

573 Veen et al., 2001) or between responses / task sets (Ebitz and Platt, 2015). The two forms

574 of conflict in our task have some intuitive similarities to the distinction between the

575 different forms of conflict in these previous studies. However, the overlap is unlikely to be

576 perfect - as Van Veen et al., showed, the flanker task can elicit both stimulus and response

577 conflict depending on the condition. Nonetheless, this study supports the conclusions

578 drawn by these previous studies - that different types of conflict may not have unitary

579 effects on brain activity.

580 Our results do not answer the important question of where the cognitive control for

581 response to conflict originally comes from. We see two possibilities, both consistent with 
582 our data. First, there may be another region - distal to dACC - that detects conflict and

583 controls responses of dACC task-selective neurons. Second, there may be no single region

584 that functions as a central executive. Certainly, it is possible to build executive systems

585 that lack a central controller (Eisenreich et al., 2017). For example, ant colonies - a

586 canonical distributed decision-making system - show what may be described as executive

587 control, even in the absence of a central executive (Franks et al., 2002; Franks et al., 2003).

588 Future work, including modeling, will be needed to disambiguate these two hypotheses. 
590

591

592

593

594

595

596

597

598

599

600

601

602

603

604

605

606

607

608

609

610

611

612

613

614

615

616

617

618

619

620

621

622

623

624

625

626

627

628

629

630

631

632

\section{METHODS}

\section{Subjects and ethics statement}

We studied two cohorts of subjects. Cohort 1 consisted of 7 patients ( 1 female) with medically refractory epilepsy who were undergoing intracranial monitoring to identify seizure onset regions. Before the start of the study, these subjects were implanted with stereo-encephalography (sEEG) depth electrodes using standard stereotactic techniques. One or more of the sEEG electrodes in this cohort spanned dorsolateral prefrontal cortex (dlPFC) to dorsal anterior cingulate cortex (dACC; Brodmann's areas 24a/b/c and 32), providing LFP recordings from both regions, as well as single unit recordings in dACC (see below; Data Acquisition).

Cohort 2 consisted of 9 patients: 8 ( 2 female) with movement disorders (Parkinson's disease or essential tremor) who were undergoing deep brain stimulation (DBS) surgery, and one male patient with epilepsy undergoing intracranial seizure monitoring. The entry point for the trajectory of the DBS electrode is typically in the inferior portion of the superior frontal gyrus or superior portion of the middle frontal gyrus, within $2 \mathrm{~cm}$ of the coronal suture. This area corresponds to dlPFC (Brodmann's areas 9 and 46). The single epilepsy patient in this cohort underwent a craniotomy for placement of subdural grid/strip electrodes in a prefrontal area including dlPFC.

All decisions regarding sEEG and DBS trajectories and craniotomy location were made solely based on clinical criteria. The Columbia University Medical Center Institutional Review Board approved these experiments, and all subjects provided informed consent prior to participating in the study.

\section{Behavioral Task}

All subjects performed the multi-source interference task (MSIT; Figure 1A). In this task, each trial began with a 500-millisecond fixation period. This was followed by a cue indicating the correct response as well as the distractor response. The cue consisted of three integers drawn from $\{0,1,2,3\}$. One of these three numbers (the "correct response cue") was different from the other two numbers (the "distractor response cues"). Subjects were instructed to indicate the identity of the correct response number on a 3-button pad. The three buttons on this pad corresponded to the numbers 1 (left button), 2 (middle) and 3 (right), respectively.

The MSIT task therefore presented two types of conflict. Simon (motor spatial) conflict occurred if the correct response cue was located in a different position in the cue than the corresponding position on the 3-button pad (e.g. ' 001 '; target in right position, but left button is correct choice). Eriksen (flanker) conflict occurred if the distractor numbers were possible button choices (e.g. '3 2 3', in which " 3 " corresponds to a possible button choice; vs. '0 20 ', in which " 0 ” does not correspond to a possible button choice).

After each subject registered his or her response, the cue disappeared and feedback appeared. The feedback consisted of the target number, but it appeared in a different color. The duration of the feedback was variable (300 to 800 milliseconds, drawn from a uniform 
633

634

635

636

637

638

639

640

641

642

643

644

645

646

647

648

649

650

651

652

653

654

655

656

657

658

659

660

661

662

663

664

665

666

667

668

669

670

671

672

673

674

675 distribution therein). The inter-trial interval varied uniformly randomly between 1 and 1.5 seconds.

The task was presented on a computer monitor controlled by the Psychophysics Matlab Toolbox (www.psychtoolbox.org; The MathWorks, Inc). This software interfaced with data acquisition cards (National Instruments,) that allowed for synchronization of behavioral events and neural data with sub-millisecond precision.

\section{Data Acquisition and preprocessing}

Single unit activity (SUA) was recorded from microelectrodes using 3 different techniques. In Cohort 1, the dlPFC-dACC sEEG electrodes were Behnke-Fried macromicro electrodes (AdTech Medical). These electrodes consist of a standard clinical depth macroelectrode shaft with a bundle of eight shielded microwires that protrude $\sim 4 \mathrm{~mm}$ from the tip (IRB-AAAB6324). These 8 microwires are referenced to a ninth unshielded microwire.

Cohort 2 provided dlPFC SUA, although it used a combination of two techniques. The DBS surgeries were performed according to standard clinical procedure, using clinical microelectrode recording (Frederick Haer Corp.). Prior to inserting the guide tubes for the clinical recordings, we placed the microelectrodes in the cortex under direct vision to record from dlPFC, (IRB-AAAK2104). The epilepsy implant in Cohort 2 included a Utahstyle microelectrode array (UMA) implanted in dlPFC (IRB-AAAB6324). In all cases, data were amplified, high-pass filtered, and digitized at 30 kilosamples per second on a neural signal processor (Blackrock Microsystems, LLC).

SUA data were re-thresholded offline at negative four times the root mean square of the $250 \mathrm{~Hz}$ high-pass filtered signal. Well-isolated action potential waveforms were then segregated in a semi-supervised manner using the T-distribution expectation-maximization method on a feature space comprised of the first three principal components using Offline Sorter (OLS) software (Plexon Inc, Dallas, TX; USA). The times of threshold crossing for identified single units were retained for further analysis.

Additive effects of Simon and Ericksen conflict. We determined what effect the combination of Ericksen and Simon conflict had on dACC activity by comparing the fit of the following three generalized linear models. First, we considered a 4-parameter "full model", which independently measured the contribution of Ericksen conflict $\left(\mathrm{C}^{\mathrm{E}}\right.$; coded as 1 when the correct response and Ericksen distractor response were in conflict, 0 otherwise), Simon conflict $\left(\mathrm{C}^{\mathrm{S}}\right)$, and the combination of both $\left(\mathrm{C}^{\mathrm{B}}\right.$; coded as 1 if and only if $\mathrm{C}^{\mathrm{E}}$ and $\mathrm{C}^{\mathrm{S}}$ were both true). This model 1) made no assumptions about the relative effects of Ericksen and Simon conflict and 2) also allowed for superadditive or subadditive effects when both forms of conflict co-occurred.

\section{$F R \sim \beta_{0}+\beta_{1} C^{E}+\beta_{2} C^{S}+\beta_{3} C^{B}$}

For the second model, the "independent model", we dropped the sub-/super-additive term, leaving a simplified, 3-parameter model. This model would be a sufficient explanation for 
676

677

678

679

680

681

682

683

684

685

686

687

688

689

690

691

692

693

694

695

696

697

698

699

700

701

702

703

the data if the dACC response to the combination of Ericksen and Simon conflict was simply the sum of the two types of conflict independently.

\section{$F R \sim \beta_{0}+\beta_{1} C^{E}+\beta_{2} C^{S}$}

Finally, in the third, "additive model", we dropped the term that allowed Simon and Ericksen conflict to have different effects (i.e. we assumed that $\beta_{1}=\beta_{2 \text { in our previous }}$ model), leaving a 2-parameter model. This model would be a sufficient explanation for the data if Ericksen and Simon conflict have both identical and additive effects on the dACC population.

\section{$F R \sim \beta_{0}+\beta_{1}\left(C^{E}+C^{S}\right)$}

We used standard model comparison (Burnham \& Andersen, 2010) to determine whether each simplifying assumption could be made with no loss of information. Models were fit to z-scored firing rates that were condition-averaged within neurons ( 9 data points per neuron, reflecting all combinations of the 3 correct response, 3 Ericksen distractors, and 3 Simon distractors) and offset terms were included for each neuron (number of neurons-1 offset terms), though the z-scoring ensured that the results did not depend on including cell identity terms.

Table S1. Additive effects of Simon and Ericksen conflict.

\begin{tabular}{|r|c|c|c|c|c|}
\hline Model: & $\boldsymbol{\beta}_{\mathbf{1}}$ & $\boldsymbol{\beta}_{\mathbf{2}}$ & $\boldsymbol{\beta}_{\mathbf{3}}$ & Likelihood & $\begin{array}{c}\text { AIC (weight) } \\
\text { BIC (weight) }\end{array}$ \\
\hline \multirow{2}{*}{ full } & $\begin{array}{c}0.029, \\
p>0.2\end{array}$ & $\begin{array}{c}0.020, \\
p>0.4\end{array}$ & $\begin{array}{c}0.012, \\
p>0.7\end{array}$ & -2313.35 & $\begin{array}{c}4920.7(0.15) \\
5824.2(0.0003)\end{array}$ \\
\hline \multirow{2}{*}{ independent } & $\begin{array}{c}0.037, \\
\mathbf{p}<\mathbf{0 . 0 2}\end{array}$ & $\begin{array}{c}0.028, \\
\mathrm{p}=0.06\end{array}$ & - & -2313.41 & $\begin{array}{c}4918.8(0.40) \\
5834.6(0.017)\end{array}$ \\
\hline \multirow{2}{*}{ additive } & $\begin{array}{c}0.033, \\
\mathbf{p}<\mathbf{0 . 0 0 3}\end{array}$ & - & - & -2313.48 & $\begin{array}{c}\mathbf{4 9 1 7 . 0 ( 1 )} \\
\mathbf{5 8 2 6 . 5}(\mathbf{1})^{*}\end{array}$ \\
\hline
\end{tabular}

* significant improvement in model fit by this metric

Task, distractor, and conflict tuning. To determine how frequently correct response, distractor response, and conflict tuning co-occurred within individual cells, we used the following ANOVA:

$$
\begin{aligned}
F R_{i j k} \sim \mu+T_{i}+D_{j}^{E}+D_{k}^{S}+ \\
C^{E}(i \neq j)+C^{S}(i \neq k)+\epsilon_{i j k}
\end{aligned}
$$


Where $\mathrm{FR}_{\mathrm{ijk}}$ is the average firing rate of the cell for the ith correct response, with the jth Ericksen distractor response, and kth Simon distractor response. Here, the T term models the effect of correct responses on neural activity, the $\mathrm{D}^{\mathrm{E}}$ and $\mathrm{D}^{\mathrm{S}}$ terms model, respectively, the effects of Ericksen and Simon distractor responses, and the $\mathrm{C}^{\mathrm{E}}$ and $\mathrm{C}^{\mathrm{S}}$ terms model the effects of conflict, meaning the mismatch between correct and distractor responses for Ericksen and Simon distractors, respectively.

Residuals. In the epiphenomenal hypothesis, conflict signals are an emergent consequence of co-activating pools of neurons that are tuned for different responses (Figure 4A). This implies that we should be able to predict activity in conflict conditions from the activity under different task and distractor conditions. Systematic deviations from these predicted values would indicate some pattern that could not have emerged because of summed activity due to task and distractor activations without some form of systematic nonlinearity (which we have no reason to expect a priori in dACC). Within each neuron, we calculated the expected firing rate for each task condition, marginalizing over distractors, and for each distractor, marginalizing over tasks. Then we estimated the expected activity for each combination of task and distractor by summing these estimates for task and distractor (Figure 3C). Subtracting this expectation from the observed pattern of activity left the residual activity that could not be explained by the linear co-activation of task and distractor conditions.

Pseudopopulations. To estimate how conflict affected neuronal population activity, we generated pseudopopulations by combining the activity of neurons that were recorded largely separately (Churchland et al., 2012; Machens et al., 2010; Mante et al., 2013; Meyers et al., 2008; Ebitz et al., 2019; Sleezer et al., 2016). Within each task condition (combination of correct response and distractor response), firing rates from separately recorded neurons were randomly drawn with replacement to create a pseudotrial firing rate vector for that task condition, with each entry corresponding to the activity of one neuron in that condition. Pseudotrial vectors were then stacked into the trials-by-neurons pseudopopulation matrix. Nineteen pseudotrials were drawn for each condition, based on the observation that a minimum of $75 \%$ of conditions had at least this number of observations, though results were identical for different choices of this value ( \pm 5 trials). All effects were confirmed via bootstrap tests across 1000 randomly re-seeded pseudopopulations. In addition, some analyses are reported with a "representative" pseudopopulation. This was the pseudopopulation that most closely matched the average condition means across 1000 random samples (i.e. the pseudopopulation seed that minimized root mean squared error from the vector of condition projection averages). These analyses focus on Eriksen conflict because this form of conflict had the larger effect on response time and caused a significant increase in the average activity of dACC neurons. Similar results were obtained for Simon conflict (data not shown).

Targeted dimensionality reduction. To determine how conflict affected population activity along task-coding dimensions, we used a form of targeted dimensionality reduction based on multinomial logistic regression (Ebitz, et al., 2018; Peixoto et al., 
2018). Targeted dimensionality reduction is a class of methods for re-representing highdimensional neural activity in a small number of dimensions that correspond to variables of interest in the data (Cohen and Maunsell, 2010; Cunningham and Yu, 2014; Mante et al., 2013; Peixoto et al., 2018; Ebitz et al., 2019). Thus, unlike principle component analysis - which reduces the dimensionality of neural activity by projecting it onto the axes that capture the most variability in the data-targeted dimensionality reduction reduces dimensionality projecting activity onto axes that encode task information or predict behavior.

Here, we were interested in how conflict changed task coding, so we first identified the axes in neural activity discriminated the three correct responses. We used multinomial logistic regression to find the separating hyperplanes in neuron-dimensional space that best separated the neural activity for one correct response (i.e. button press 1) from activity during the other correct responses (i.e. not button press 1). Formally, we fit a system of three logistic classifiers:

$$
\log \left(\frac{p(\text { choice }=i \mid \mathrm{X})}{1-p(\text { choice }=i \mid \mathrm{X})}\right)=\mathrm{X} \beta_{i}
$$

Where $\mathrm{X}$ is the trials by neurons pseudopopulation matrix of firing rates and $\beta \mathrm{i}$ is the vector of coefficients that best differentiated neural activity on trials in which a choice matching category $i$ is chosen from activity on other trials (fit via regularized maximum likelihood). The separating hyperplane for each choice $\mathrm{i}$ is the vector (a) that satisfies:

$$
a^{T} \beta_{i}=0
$$

Meaning that $\beta \mathrm{i}$ is a vector orthogonal to the separating hyperplane in neurondimensional space, along which position is proportional to the log odds of that correct response: this is the the coding dimension for that correct response. By projecting a pseudotrial vector $\mathrm{x}$ onto this coding dimension, we are essentially re-representing the trial in terms of its distance from the separating hyperplane corresponding to task i. Projecting that trial onto all three classifiers, then re-represents that high-dimensional pseudotrial in three dimensions - each one of which corresponds to the coding dimension of a different response.

We used an identical approach to identify the coding dimensions corresponding to each distractor. To identify coding dimensions corresponding to task conditions (combination of 3 correct responses and 3 distractor responses, if present), we used the same approach to classify the 12 task conditions. 


\section{REFERENCES}

Alexander, W. H., \& Brown, J. W. (2011). Medial prefrontal cortex as an action-outcome predictor. Nature neuroscience, 14(10), 1338.

Amiez C, Joseph JP, Procyk E. 2005. Anterior cingulate error-related activity is modulated by predicted reward. Eur. J. Neurosci. 21:3447-52

Amiez C, Joseph JP, Procyk E. 2006. Reward encoding in the monkey anterior cingulate cortex. Cereb. Cortex 16:1040-55

Azab, H., \& Hayden, B. Y. (2017). Correlates of decisional dynamics in the dorsal anterior cingulate cortex. PLoS biology, 15(11), e2003091.

Blanchard, T. C., Piantadosi, S. T., \& Hayden, B. Y. (2018). Robust mixture modeling reveals category-free selectivity in reward region neuronal ensembles. Journal of neurophysiology, 119(4), 1305-1318.

Blanchard TC, Hayden BY. 2014. Neurons in dorsal anterior cingulate cortex signal postdecisional variables in a foraging task. J. Neurosci. 34:646-55

Botvinick, M. M., Braver, T. S., Barch, D. M., Carter, C. S., \& Cohen, J. D. (2001). Conflict monitoring and cognitive control. Psychological review, 108(3), 624.

Botvinick, M., Nystrom, L. E., Fissell, K., Carter, C. S. \& Cohen, J. D. Conflict monitoring versus selection-for-action in anterior cingulate cortex. Nature 402, 179-181 (1999).

Botvinick, M. M. \& Cohen, J. D. The Computational and Neural Basis of Cognitive Control: Charted Territory and New Frontiers. Cogn. Sci. 38, 1249-1285 (2014).

Botvinick, M. \& Braver, T. Motivation and Cognitive Control: From Behavior to Neural Mechanism. Annu. Rev. Psychol. 66, 83-113 (2015).

Bryden, D. W., Brockett, A. T., Blume, E., Heatley, K., Zhao, A., \& Roesch, M. R. (2018). Single neurons in anterior cingulate cortex signal the need to change action during performance of a stop-change task that induces response competition. Cerebral Cortex, 29(3), 1020-1031.

Burnham, K. P., \& Anderson, D. R. (2010). Model selection and multimodel inference: a practical information-theoretic approach. Springer Science \& Business Media.

Bush, G., Luu, P., \& Posner, M. I. (2000). Cognitive and emotional influences in anterior cingulate cortex. Trends in cognitive sciences, 4(6), 215-222.

Cai X, Padoa-Schioppa C. 2012. Neuronal encoding of subjective value in dorsal and ventral anterior cingulate cortex. J. Neurosci. 32:3791-808

Churchland, M. M., Cunningham, J. P., Kaufman, M. T., Foster, J. D., Nuyujukian, P., Ryu, S. I., \& Shenoy, K. V. (2012). Neural population dynamics during reaching. Nature, 487(7405), 51.

Cisek P and Kalaska JF (2010) Neural mechanisms for interacting with a world full of action choices. Annual Review of Neuroscience 33: 269-298.

Cisek, P. (2012). Making decisions through a distributed consensus. Current opinion in neurobiology, 22(6), 927-936.

Cisek, P. (2007). Cortical mechanisms of action selection: the affordance competition hypothesis. Philosophical Transactions of the Royal Society B: Biological Sciences, 362(1485), 1585-1599. 
832

833

834

835

836

837

838

839

840

841

842

843

844

845

846

847

848

849

850

851

852

853

854

855

856

857

858

859

860

861

862

863

864

865

866

867

868

869

870

871

872

873

874

875

Cohen, M. R., \& Maunsell, J. H. (2010). A neuronal population measure of attention predicts behavioral performance on individual trials. Journal of Neuroscience, 30(45), 15241-15253.

Cole, M. W., Yeung, N., Freiwald, W. A., \& Botvinick, M. (2009). Cingulate cortex: diverging data from humans and monkeys. Trends in neurosciences, 32(11), 566574.

Cole, M. W., Yeung, N., Freiwald, W. A., \& Botvinick, M. (2010). Conflict over cingulate cortex: between-species differences in cingulate may support enhanced cognitive flexibility in humans. Brain, behavior and evolution, 75(4), 239-240.

Cunningham, J. P., \& Yu, B. Y. (2014). Dimensionality reduction for large-scale neural recordings. Nature neuroscience, 17(11), 1500.

David, S. V., \& Hayden, B. Y. (2012). Neurotree: A collaborative, graphical database of the academic genealogy of neuroscience. PloS one, 7(10).

Desimone, R., \& Duncan, J. (1995). Neural mechanisms of selective visual attention. Annual review of neuroscience, 18(1), 193-222.

Desimone, R. (1996). Neural mechanisms for visual memory and their role in attention. Proceedings of the National Academy of Sciences, 93(24), 13494-13499.

Ebitz, R. B., \& Hayden, B. Y. (2016). Dorsal anterior cingulate: a Rorschach test for cognitive neuroscience. Nature neuroscience, 19(10), 1278.

Ebitz, R. B., \& Moore, T. (2017). Selective modulation of the pupil light reflex by microstimulation of prefrontal cortex. Journal of Neuroscience, 37(19), 5008-5018.

Ebitz, R. B., \& Platt, M. L. (2015). Neuronal activity in primate dorsal anterior cingulate cortex signals task conflict and predicts adjustments in pupil-linked arousal. Neuron, 85(3), 628-640.

Ebitz, R. B., Tu, J. C., \& Hayden, B. Y. (2019). Rule adherence warps decision-making. bioRxiv.

Ebitz, R. B., Albarran, E., \& Moore, T. (2018). Exploration disrupts choice-predictive signals and alters dynamics in prefrontal cortex. Neuron, 97(2), 450-461.

Eisenreich, B. R., Akaishi, R., \& Hayden, B. Y. (2017). Control without controllers: toward a distributed neuroscience of executive control. Journal of cognitive neuroscience, 29(10), 1684-1698.

Egner, T., \& Hirsch, J. (2005). Cognitive control mechanisms resolve conflict through cortical amplification of task-relevant information. Nature neuroscience, 8(12), 1784.

Farashahi, S., Azab, H., Hayden, B., \& Soltani, A. (2018). On the flexibility of basic risk attitudes in monkeys. Journal of Neuroscience, 38(18), 4383-4398.

Finkelstein, A., Fontolan, L., Economo, M. N., Li, N., Romani, S., \& Svoboda, K. (2019). Attractor dynamics gate cortical information flow during decision-making. bioRxiv.

Franks, N. R., Dornhaus, A., Fitzsimmons, J. P., \& Stevens, M. (2003). Speed versus accuracy in collective decision making. Proceedings of the Royal Society of London, Series B, Biological Sciences, 270, 2457-2463.

Franks, N. R., Pratt, S. C., Mallon, E. B., Britton, N. F., \& Sumpter, D. J. (2002). Information flow, opinion polling and collective intelligence in house-hunting 
social insects. Philosophical Transactions of the Royal Society of London, Series B, Biological Sciences, 357, 1567-1583.

Gemba H, Sasaki K, Brooks V. 1986. 'Error' potentials in limbic cortex (anterior cingulate area 24) of monkeys during motor learning. Neurosci. Lett. 70:223-27

Hayden, B. Y., Pearson, J. M., \& Platt, M. L. (2011). Neuronal basis of sequential foraging decisions in a patchy environment. Nature neuroscience, 14(7), 933.

Hayden, B. Y. (2019). Why has evolution not selected for perfect self-control? Philosophical Transactions of the Royal Society B, 374(1766), 20180139.

Heilbronner, S. R., \& Hayden, B. Y. (2016). Dorsal anterior cingulate cortex: a bottom-up view. Annual review of neuroscience, 39, 149-170.

Heilbronner, S. R., \& Hayden, B. Y. (2016). The description-experience gap in risky choice in nonhuman primates. Psychonomic bulletin \& review, 23(2), 593-600.

Hillman KL, Bilkey DK. 2010. Neurons in the rat anterior cingulate cortex dynamically encode cost-benefit in a spatial decision-making task. J. Neurosci. 30:7705-13

Hunt, L. T., \& Hayden, B. Y. (2017). A distributed, hierarchical and recurrent framework for reward-based choice. Nature Reviews Neuroscience, 18(3), 172.

Hunt, L. T., Malalasekera, W. N., de Berker, A. O., Miranda, B., Farmer, S. F., Behrens, T. E., \& Kennerley, S. W. (2018). Triple dissociation of attention and decision computations across prefrontal cortex. Nature neuroscience, 21(10), 1471.

Isomura Y, Ito Y, Akazawa T, Nambu A, Takada M. 2003. Neural coding of "attention for action" and "response selection" in primate anterior cingulate cortex. J. Neurosci. 23:8002-12

Ito S, Stuphorn V, Brown JW, Schall JD. 2003. Performance monitoring by the anterior cingulate cortex during saccade countermanding. Science 302:120-22

Johnston, K., Levin, H. M., Koval, M. J., \& Everling, S. (2007). Top-down control-signal dynamics in anterior cingulate and prefrontal cortex neurons following task switching. Neuron, 53(3), 453-462.

Kennerley SW, Behrens TE, Wallis JD. 2011. Double dissociation of value computations in orbitofrontal and anterior cingulate neurons. Nat. Neurosci. 14:1581-89

Kerns, J. G., Cohen, J. D., MacDonald, A. W., Cho, R. Y., Stenger, V. A., \& Carter, C. S. (2004). Anterior cingulate conflict monitoring and adjustments in control. Science, 303(5660), 1023-1026.

Kolling, N., Wittmann, M. K., Behrens, T. E., Boorman, E. D., Mars, R. B., \& Rushworth, M. F. (2016). Value, search, persistence and model updating in anterior cingulate cortex. Nature neuroscience, 19(10), 1280.

Ma, L., Chan, J. L., Johnston, K., Lomber, S. G., \& Everling, S. (2019). Macaque anterior cingulate cortex deactivation impairs performance and alters lateral prefrontal oscillatory activities in a rule-switching task. PLoS biology, 17(7), e3000045.

MacDonald, A. W., Cohen, J. D., Stenger, V. A., \& Carter, C. S. (2000). Dissociating the role of the dorsolateral prefrontal and anterior cingulate cortex in cognitive control. Science, 288(5472), 1835-1838.

Machens, C. K., Romo, R., \& Brody, C. D. (2010). Functional, but not anatomical, separation of "what" and "when" in prefrontal cortex. Journal of Neuroscience, 30(1), 350-360. 
Mansouri, F. A., Egner, T., \& Buckley, M. J. (2017). Monitoring demands for executive control: shared functions between human and nonhuman primates. Trends in neurosciences, 40(1), 15-27.

Mante V, Sussillo D, Shenoy KV, Newsome WT. Context-dependent computation by recurrent dynamics in prefrontal cortex. Nature 503: 78 - 84, 2013. doi:10.1038/nature12742.

Meyers, E. M., Freedman, D. J., Kreiman, G., Miller, E. K., \& Poggio, T. (2008). Dynamic population coding of category information in inferior temporal and prefrontal cortex. Journal of neurophysiology, 100(3), 1407-1419.

McAdams, C. J., \& Maunsell, J. H. (1999). Effects of attention on orientation-tuning functions of single neurons in macaque cortical area V4. Journal of Neuroscience, 19(1), 431-441.

Michelet, T., Duncan, G. H., \& Cisek, P. (2010). Response competition in the primary motor cortex: corticospinal excitability reflects response replacement during simple decisions. Journal of neurophysiology, 104(1), 119-127.

Michelet T, Bioulac B, Langbour N, Goillandeau M, Guehl D, Burbaud P. 2015. Electrophysiological correlates of a versatile executive control system in the monkey anterior cingulate cortex. Cereb. Cortex 26:1684-97

Nakamura K, Roesch MR, Olson CR. 2005. Neuronal activity in macaque SEF and ACC during performance of tasks involving conflict. J. Neurophysiol. 93:884-908

Pastor-Bernier A and Cisek P (2011) Neural correlates of biased competition in premotor cortex. The Journal of Neuroscience: The Official Journal of the Society for Neuroscience 31(19): 7083-7088.

Paus, T. (2001). Primate anterior cingulate cortex: where motor control, drive and cognition interface. Nature reviews neuroscience, 2(6), 417.

Peixoto, D., Verhein, J. R., Kiani, R., Kao, J. C., Nuyujukian, P., Chandrasekaran, C., Brown. J, Fong, S., Ryu, S. I., Shenoy, K. V., and Newsome, W. T. (2019). Decoding and perturbing decision states in real time. bioRxiv 681783; doi: https://doi.org/10.1101/681783

Pezzulo, G., \& Cisek, P. (2016). Navigating the affordance landscape: feedback control as a process model of behavior and cognition. Trends in cognitive sciences, 20(6), 414-424.

Pirrone, A., Azab, H., Hayden, B. Y., Stafford, T., \& Marshall, J. A. (2018). Evidence for the speed-value trade-off: Human and monkey decision making is magnitude sensitive. Decision, 5(2), 129.

Schall, J. D., \& Emeric, E. E. (2010). Conflict in Cingulate Cortex Function between Humans and Macaque Monkeys: More Apparent than Real: Commentary on Cole MW, Yeung N, Freiwald WA, Botvinick M (2009): Cingulate Cortex: Diverging Data from Humans and Monkeys. Trends Neurosci 32: 566-574. Brain, behavior and evolution, 75(4), 237.

Shenhav, A., Botvinick, M. M., \& Cohen, J. D. (2013). The expected value of control: an integrative theory of anterior cingulate cortex function. Neuron, 79(2), 217-240. 
Shenhav, A., Musslick, S., Lieder, F., Kool, W., Griffiths, T. L., Cohen, J. D., \& Botvinick, M. M. (2017). Toward a rational and mechanistic account of mental effort. Annual review of neuroscience, 40, 99-124.

Shenhav, A., Cohen, J. D., \& Botvinick, M. M. (2016). Dorsal anterior cingulate cortex and the value of control. Nature neuroscience, 19(10), 1286.

Sheth SA, Mian MK, Patel SR, Asaad WF, Williams ZM, et al. 2012. Human dorsal anterior cingulate cortex neurons mediate ongoing behavioural adaptation. Nature 488:218-21

Sleezer, B. J., Castagno, M. D., \& Hayden, B. Y. (2016). Rule encoding in orbitofrontal cortex and striatum guides selection. Journal of Neuroscience, 36(44), 1122311237.

Smith, E. H., Horga, G., Yates, M. J., Mikell, C. B., Banks, G. P., Pathak, Y. J., ... \& Sheth, S. A. (2019). Widespread temporal coding of cognitive control in the human prefrontal cortex. Nature neuroscience, 1-9.

Strait CE, Sleezer BJ, Blanchard TC, et al. (2016) Neuronal selectivity for spatial positions of offers and choices in five reward regions. Journal of Neurophysiology 115(3): 1098-1111.

Stuphorn, V., Taylor, T. L., \& Schall, J. D. (2000). Performance monitoring by the supplementary eye field. Nature, 408(6814), 857.

Suzuki, M., \& Gottlieb, J. (2013). Distinct neural mechanisms of distractor suppression in the frontal and parietal lobe. Nature neuroscience, 16(1), 98.

Van Veen, V., Cohen, J. D., Botvinick, M. M., Stenger, V. A., \& Carter, C. S. (2001). Anterior cingulate cortex, conflict monitoring, and levels of processing. Neuroimage, 14(6), 1302-1308.

Widge, A. S., Zorowitz, S., Basu, I., Paulk, A. C., Cash, S. S., Eskandar, E. N., ... \& Dougherty, D. D. (2019a). Deep brain stimulation of the internal capsule enhances human cognitive control and prefrontal cortex function. Nature communications, 10(1), 1-11.

Widge, A. S., Heilbronner, S. R., \& Hayden, B. Y. (2019b). Prefrontal cortex and cognitive control: new insights from human electrophysiology. F1000Research, 8.

Yoo SBM and Hayden BY (2018) Economic choice as an untangling of options into actions. Neuron 99(3): 434-447. 